\title{
Evaluation of the BD Phoenix CPO detect panel for prediction of Ambler class carbapenemases
}

\author{
Daniel Jonas ${ }^{1 \bowtie}$, Sandra Reuter ${ }^{1}$, Sarah Klassen ${ }^{1}$, Sabine Weber ${ }^{1}$, Marion Buck ${ }^{1}$, \\ Tommaso Giani ${ }^{2,3}$, Gian Maria Rossolini ${ }^{2,3}$ \& Hajo Grundmann ${ }^{1}$
}

Rapid detection of carbapenemases as a cause of resistance is beneficial for infection control and antimicrobial therapy. The BD Phoenix NMIC-502 panel and CPO detect test identifies presence of carbapenemases in Enterobacterales such as Klebsiella pneumoniae and assigns them to Ambler classes. To evaluate the performance of the CPO detect panel, we employed a European collection of $1222 \mathrm{~K}$. pneumoniae including carbapenem non-susceptible and susceptible clinical isolates from 26 countries, for which draft genomes were available after Illumina sequencing and the presence of carbapenemase genes had been identified by ARIBA gene calling. The CPO panel detected 488 out of 494 carbapenemase-encoding isolates as positive and six as negative. One-hundred and two isolates were tested positive for carbapenemase in the absence of any carbapenemase gene. The CPO panel identified 229 out of $230 \mathrm{KPC}$-positive isolates as carbapenemase producing and classified 62 of these as class A enzyme. Similarly, the CPO panel correctly specified 167 of 182 as class D. Regarding metallo-beta-lactamases, the CPO panel assigned 78 of $90 \mathrm{MBL}$ positive isolates to class B enzymes. The sensitivity of the CPO panel in detecting carbapenemase activity was $99.5 \%, 97.7 \%$ and $98.3 \%$ for class $A, B$ and $D$ enzymes, respectively. The sensitivity in assignation to Ambler class A, B and D was $27 \%, 86 \%$ and $91 \%$, respectively. An overall sensitivity of $98.8 \%$ and specificity of $86 \%$ in unclassified detection of carbapenemases was observed, with frequent false positive detection of carbapenemase producing organisms, thus rendering further confirmatory tests necessary.

There has been an increasing number of reports on the spread of carbapenem-resistant Gram-negative pathogens including Enterobacterales (CRE), Gram-negative non-fermenters such as Pseudomonas aeruginosa and Acinetobacter spp., as well as carbapenemase-producing organisms (CPO) and their limited treatment options ${ }^{1-3}$.

In principle, carbapenem resistance can be caused by (i) reduced cell permeability to antibiotics due to loss of influx porins or increased efflux pump activity, possibly in combination with the production of extendedspectrum $\beta$-lactamases (ESBL) or AmpC-type $\beta$-lactamases, or by (ii) production of inactivating enzymes, i.e., carbapenemases. While the former mechanisms arise from mutations and may impair biological fitness ${ }^{4-6}$, presumably being rather more self-limiting, carbapenemases in general are of major concern, especially since they are often encoded on mobile genetic elements, which can spread rapidly by lateral gene transfer across genetic and species boundaries. Distinguishing between these two principal resistance mechanisms can therefore be both meaningful and guiding from the point of view of infection control. Moreover, detailed classification of carbapenemases can be valuable for therapy, since the new beta-lactamase inhibitor combinations have only a spectrum limited to serine carbapenemases of class A (and in some cases to class D), while only monobactams are stable to class B metallo-beta-lactamases ${ }^{7-9}$.

For this reason, there is increasing interest in the detection of carbapenemase production in the diagnostic microbiology laboratory. Several diagnostic tests have been developed to prove production of carbapenemases, e.g. the Carba NP-direct test ${ }^{10}$, the modified carbapenem inactivation method $(\mathrm{mCIM})^{11}$, or the modified Hodge test ${ }^{12,13}$. However, these tests do not differentiate between the carbapenemase class, which can be determined either by phenotypic tests using inhibitors ${ }^{14,15}$ or by tests that detect the enzymes by lateral-flow immunochromatography ${ }^{16,17}$, or the carbapenemase genes by molecular detection methods using PCR ${ }^{18,19}$, microarrays $^{20,21}$ or whole genome sequencing (WGS $)^{22-24}$. However, all these tests must be performed in addition to

${ }^{1}$ Institute for Infection Prevention and Hospital Epidemiology, Faculty of Medicine, University of Freiburg, Freiburg, Germany. ${ }^{2}$ Department of Experimental and Clinical Medicine, University of Florence, Florence, Italy. ${ }^{3} \mathrm{Clinical}$ Microbiology and Virology Unit, Florence Careggi University Hospital, Florence, Italy. ${ }^{\square}$ email: daniel.jonas@ uniklinik-freiburg.de 
conventional antimicrobial susceptibility testing (AST), and are usually carried out with carbapenem-resistant isolates after the results of AST are available, leading to some delay in the information released.

The BD Phoenix is a bacterial growth-based automated system for determination of antibiotic resistance characteristics by use of various test panels. The "CPO detect test" is embedded in the NMIC-502 panel of the BD Phoenix card for AST of Gram-negative pathogens (in short „the CPO panel”), which provides a means to (i) detect and (ii) classify carbapenemases in line with AST, and without any further workload, utilizing bacterial growth in the presence of meropenem, doripenem, temocillin and cloxacillin, both alone and in combination with various chelators and beta-lactamase inhibitors (Becton Dickinson, instructions for use of the test).

Few previous studies have investigated the performance of the CPO detect test $\mathrm{t}^{25-30}$. These studies are mainly based on smaller collections of locally or regionally collected/sampled CPO-positive strains and just some 30-50 CPO-negative control strains selected on the basis of resistance without carbapenemase activity-which has already been discussed as a limitation in assessment of test specificity.

The study presented here is a large evaluation of the performance of the CPO detection test included in the BD Phoenix ${ }^{\prime \prime}$ NMIC-502 panel for (i) detection and (ii) classification of carbapenemases. It is based on a representative European collection of over 1000 Klebsiella pneumoniae isolates from 26 countries which originated from surveillance of carbapenem resistance throughout Europe in 2014, and all of which had previously been subjected to WGS analysis ${ }^{31}$. Of note, this collection not only included carbapenem-resistant isolates but also an equal number of non-resistant isolates from microbiological routine diagnostics throughout Europe.

\section{Results}

In accordance with AST, the CPO panel can distinguish carbapenem resistances due to carbapenemase production from those mediated by other mechanisms (e. g. altered permeability) in Enterobacterales such as Klebsiella pneumoniae. The phenotypes of unspecified carbapenemases are designated CARB, whereas class A, B or D enzymes are flagged as CARBA, CARBB or CARBD. While the test only indicates one of the four possible results, the following analysis distinguishes between the detection of any carbapenemases (CARB_OR), regardless of class, and the more precise and specific assignment to one of the three classes.

For a set of $1222 \mathrm{~K}$. pneumoniae, data on the presence/absence of carbapenemase resistance genes and multilocus sequence types were available for subsequent analyses based on draft genome sequencing.

Carbapenemases belonging to three Ambler classes, namely class A (including KPC-2, -3 and -12), class B metallo-beta-lactamases (MBL of IMP-1, NDM-1, VIM-1 and - 4 type) and class D carbapenemases (including OXA-48-like [OXA 48, 162, 204, 232]) were identified by employing ARIBA gene identification by local assembly.

Of the 1222 isolates, 503 contained KPC, MBL and OXA carbapenemase genes, according to Illumina sequence data. Resolution of 15 initially discrepant results between positive sequence data and negative phenotypic CPO panel results using gene-specific PCR resulted in 9 PCR negative results and 6 strains that remained positive for carbapenemase sequences based on both the sequence data and the PCR results. In the following, 494 isolates were thus considered genotypically positive and 728 genotypically negative.

In 488 of 494 genotypic positive strains, the CPO panel detected carbapenemases irrespective of any of the four particular phenotypes (CARB, CARBA, CARBB or CARBD) (overall sensitivity 98.8\%). None of the four CPO tests detected the presence of a carbapenemase phenotype in six of 494 isolates with a carbapenemase genotype (Table 1). According to sequence data, these strains with false negative CPO test result contained sequences coding for the following carbapenemases confirmed by PCR: OXA-48-like (3), KPC3 (1), VIM-1-like (2).

Of 728 isolates without detectable carbapenemase encoding genes, the CPO test produced a negative result in 626 strains, with a specificity of $86.0 \%$. One hundred and two isolates tested phenotypically positive for carbapenemase in the absence of identification of any known carbapenemase coding genes in the draft genome data, giving an accuracy of $91.2 \%$ and a negative or positive predictive value of $99.1 \%$ or $82.7 \%$, respectively, for detection of carbapenemases. For a more discriminant analysis, especially of the discrepant test results, CPO detection results were considered in parallel with the MIC values measured for meropenem or temocillin (Table 2). According to the NMIC-502 panel AST, most (90) of these 102 (any CARB) phenotypically false positive isolates had-only a slightly/moderately increased MIC against meropenem of $0.25-8 \mathrm{mg} / \mathrm{L}$, in contrast to 342 of 488 concordantly positive isolates with an MIC of $>8 \mathrm{mg} / \mathrm{L}$ (Table 2).

The CPO panel was unable to classify 168 of 230 carbapenemase-encoding class A gene-positive isolates (Table 1). Yet, the CPO test flagged the presence of a carbapenemase phenotype without further classification (CARB) in 167 of these KPC-positive isolates (Table 3). Remarkably, nearly all of these merely CARB-positive strains exhibited a meropenem MIC of $>8 \mathrm{mg} / \mathrm{L}$ (Table 2). Sensitivity in classifying class A carbapenemases was $27.0 \%$, specificity was $98.5 \%$. A class A enzyme was indicated in the absence of a corresponding genotype in 15 of 992 isolates.

With respect to identifying the 90 class B gene positive isolates among the 1222 clinical isolates under investigation, the CPO detect panel demonstrated a sensitivity of $86.7 \%$ and a specificity of $99.1 \%$ (Table 1 ). Six class $\mathrm{B}$ gene positive, yet CARBB phenotype negative isolates were at least marked as CARB; one of these encoded class A and B carbapenemases (Table 3). Four class B positive and CARBB negative isolates were marked as CARBD-positive, whereby three of these contained Class B and class D encoding genes.

As regards the 182 class D gene positive isolates, assessment by the CPO detect panel resulted in a sensitivity of $91.8 \%$ and a specificity of $99.8 \%$. All 15 isolates except one, which was not detected as CARBD among the 182 class D gene positive isolates, showed a temocillin MIC of $>32 \mathrm{mg} / \mathrm{L}$ (Table 2). Nine and three class D positive isolates were at least flagged as CARB- and CARBB-positive (Table 3).

Few isolates encoding two different Ambler classes, A and B (1), A and D (1) and B and D (6), resulted in detection of only one unspecified carbapenemase (CARB), or classification of only one of the two carbapenemase types (Table 3). 


\begin{tabular}{|c|c|c|}
\hline \multirow[b]{2}{*}{ Carbapenemase encoding genotype/Class-(CPO carbapenemase phenotype) } & \multicolumn{2}{|c|}{ CPO detect } \\
\hline & Negative & Positive \\
\hline \multicolumn{3}{|l|}{ Any carbapenemases encoding DNA-(CARB, CARBA, CARBB OR CARBD) } \\
\hline Neg. & 626 & 102 \\
\hline Pos. & 6 & 488 \\
\hline \multicolumn{3}{|l|}{ Class A-(CARBA) } \\
\hline Neg. & 977 & 15 \\
\hline Pos. & 168 & 62 \\
\hline \multicolumn{3}{|l|}{ Class B-(CARBB) } \\
\hline Neg. & 1122 & 10 \\
\hline Pos. & 12 & 78 \\
\hline \multicolumn{3}{|l|}{ Class D-(CARBD) } \\
\hline Neg. & 1038 & 2 \\
\hline Pos. & 15 & 167 \\
\hline \multicolumn{3}{|l|}{ Any carbapenemases encoding DNA (CARB) } \\
\hline Neg. & 652 & 76 \\
\hline Pos. & 314 & 180 \\
\hline
\end{tabular}

Table 1. CPO panel for (i) detection or (ii) classification of (un-)classified carbapenemase phenotypes (columns) in 1222 genotyped strains (rows). Phenotypes have been placed in parentheses. The abundancy of phenotypical classifications (CARBA, CARBB and CARBD) in strains with genotypical classes A, B or D are detailed. For basic detection of carbapenemases regardless of their classification, CPO detect results for carbapenemase phenotypes without further details (CARB) or with any of the four possible data outputs (CARB, CARBA, CARBB or CARBD) are compared with the presence or absence of any carbapenemase encoding DNA, i.e., regardless of the Ambler classification of the gene.

In summary, the CPO panel identified isolates as producers of any carbapenemase phenotype with an overall sensitivity of $98.8 \%$ and a specificity of $86.0 \%$.

The sensitivity of the test in categorizing the phenotypic carbapenemase activity at the Ambler class level was $27.0 \%$ for class A, $86.7 \%$ for class B and $91.8 \%$ for class D. Nevertheless, 167 of 168 class A gene encoding strains without CARBA detection revealed a CARB positive phenotype result for unspecified carbapenemases. Only two, respectively three class B or D genotype positive isolates remained without a phenotypic result pointing to carbapenemases. Specificity in phenotypic classification was $98.5 \%$ for class A, $99.1 \%$ for class B, and $99.8 \%$ for class D.

\section{Discussion}

This test is designed to differentiate between carbapenemase-producing organisms and resistant isolates without carbapenemases among carbapenem-resistant Gram-negative pathogens. Furthermore, fine differentiation and specification of carbapenemases into the various Ambler classes can guide antimicrobial therapy.

Previous studies on the performance of the CPO detect panel were hampered by employing strains of mainly regional origin (e.g., Louisiana, Singapore and South of Germany). Furthermore, the small number of negative control strains limited assessment of the specificity of these tests.

Here, we employed a European collection of carbapenem susceptible and non-susceptible clinical isolates from 26 European countries to evaluate the predictive capacity of the CPO detect panel.

Due to the large number of strains assessed in the study presented here, local effects and peculiarities could be largely excluded. Finally, the study is also based on a large collection of carefully collected positive as well as negative strains, which were scrutinized by WGS rather than by phenotypic microbiological comparison techniques.

All previous studies found a high sensitivity of $90-100 \%$ in the detection of carbapenemase, regardless of its classification. Our study confirmed the high sensitivity (98.8\%) exhibited in detecting any carbapenemase; this is also consistent with the manufacturer's specifications of $98.3 \%$ positive agreement.

However, earlier studies found a low specificity of only 55-68\%, i.e., a high rate of false positives, in contrast to the information from the manufacturer of a high negative agreement of over $95 \%$ compared to several different comparative methods. Based on a larger number of 728 characterized strains lacking carbapenemase encoding genes, we found a higher specificity of $86.0 \%$; however, this is still limiting in practical application. Therefore, others have proposed conducting additional confirmatory rapid CPO tests to detect positive strains, such as the Carba NP test, although this is of limited practical use in daily routine. Of note, most false positive reported strains had an MIC against meropenem of 1-8 mg/L, which could lead to false conclusions regarding infection control. In $50 \%$ of cases, broad spectrum beta-lactamases and/or a loss of porins could be detected genetically (data not shown), which was also discussed in another similar study as a source of possible undercount bias ${ }^{28}$. Due to the large number of strains of diverse European origin local effects and peculiarities could be largely excluded.

Regarding the test performance in terms of assignment of carbapenemases to Ambler classes, an overall accuracy of over $95 \%$ is reported by the manufacturer, without further details given on the actual proportion of the three different classes evaluated. Different results have been reported by others. 


\begin{tabular}{|c|c|c|c|c|c|c|c|c|c|c|c|c|c|c|c|c|c|c|}
\hline \multirow{3}{*}{$\begin{array}{l}\text { Any carbape- } \\
\text { nemase } \\
\text { genotype }\end{array}$} & \multicolumn{18}{|c|}{ Any CPO carbapenemase (CARB, CARBA, CARBB or CARBD) detected } \\
\hline & \multicolumn{9}{|c|}{ None } & \multicolumn{8}{|l|}{ Any } & \multirow[b]{2}{*}{ Sum } \\
\hline & $\leq 0.125$ & 0.25 & 0.5 & 1 & 2 & 4 & 8 & $>8$ & $\begin{array}{l}\text { Sum of none } \\
\text { detected }\end{array}$ & 0.25 & 0.5 & 1 & 2 & 4 & 8 & $>8$ & $\begin{array}{l}\text { Sum of any } \\
\text { detected }\end{array}$ & \\
\hline Any & 2 & & 1 & & 1 & & & 2 & 6 & 5 & 19 & 17 & 45 & 41 & 19 & 342 & 488 & 494 \\
\hline Sum & 583 & 28 & 6 & 5 & 5 & 3 & & 2 & 632 & 13 & 28 & 26 & 67 & 66 & 36 & 354 & 590 & 1222 \\
\hline \multirow{2}{*}{$\begin{array}{l}\text { Any carbape- } \\
\text { nemase } \\
\text { genotype }\end{array}$} & \multicolumn{8}{|c|}{ Meropenem MIC (CPO tested) } & & Merop & enem & MIC & O t & & & & & \multirow[b]{2}{*}{ Sum } \\
\hline & $\leq 0.125$ & 0.25 & 0.5 & 1 & 2 & 4 & 8 & $>8$ & absent & 0.25 & 0.5 & 1 & 2 & 4 & 8 & $>8$ & present & \\
\hline None & 581 & 35 & 10 & 10 & 7 & 5 & 1 & 3 & 652 & 1 & 4 & 4 & 19 & 23 & 16 & 9 & 76 & 728 \\
\hline Any & 2 & 3 & 17 & 16 & 44 & 41 & 19 & 172 & 314 & 2 & 3 & 1 & 2 & & & 172 & 180 & 494 \\
\hline Sum & 583 & 38 & 27 & 26 & 51 & 46 & 20 & 175 & 966 & 3 & 7 & 5 & 21 & 23 & 16 & 181 & 256 & 1222 \\
\hline Neg. & 583 & 34 & 31 & 29 & 69 & 67 & 32 & 132 & 977 & 6 & 3 & 2 & 2 & & & 2 & 15 & 992 \\
\hline Pos. & & 1 & & & 1 & & & 166 & 168 & & & & & 2 & 4 & 56 & 62 & 230 \\
\hline Sum & 583 & 35 & 31 & 29 & 70 & 67 & 32 & 298 & 1145 & 6 & 3 & 2 & 2 & 2 & 4 & 58 & 77 & 1222 \\
\hline & CPO clas & $s \mathrm{AOR}$ & unsp & car & penen & se $(\mathrm{CA}$ & RBA C & CAR & B) detected & & & & & & & & & \\
\hline & CARBA & OR CA & $2 B$ ab & & & & & & & CARB & A OR & CARI & reser & & & & & \\
\hline Class A & Meropen & em MI & $(\mathrm{CP}$ & teste & & & & & & Merop & enem & MIC & O t & & & & & \\
\hline genotype & $\leq 0.125$ & 0.25 & 0.5 & 1 & 2 & 4 & 8 & $>8$ & or CARB absent & \begin{tabular}{|l|l|}
0.25 \\
\end{tabular} & 0.5 & 1 & 2 & 4 & 8 & $>8$ & or CARB present & Sum \\
\hline \begin{tabular}{l|l} 
Neg. \\
\end{tabular} & 583 & 32 & 24 & 24 & 48 & 44 & 16 & 117 & \begin{tabular}{l|l}
888 \\
\end{tabular} & 8 & 10 & 7 & 23 & 23 & 16 & 17 & 104 & 992 \\
\hline Pos. & & & & & 1 & & & & 1 & 1 & & & & 2 & 4 & 222 & 229 & 230 \\
\hline Sum & 583 & 32 & 24 & 24 & 49 & 44 & 16 & 117 & 889 & 9 & 10 & 7 & 23 & 25 & 20 & 239 & 333 & 1222 \\
\hline & CPO clas & $s$ D car & bapen & mase & CARB & detec & & & & & & & & & & & & \\
\hline & CARBD & absent & & & & & & & & CARB & D pre & & & & & & & Sum \\
\hline $\begin{array}{l}\text { Class D } \\
\text { carbape-nemase }\end{array}$ & Meropen & em MI & $\mathrm{C}(\mathrm{CP}$ & teste & & & & & & Merop & enem & MIC & PO te & & & & & \\
\hline genotype & $\leq 0.125$ & 0.25 & 0.5 & 1 & 2 & 4 & 8 & $>8$ & absent & \begin{tabular}{|l|l|}
0.25 \\
\end{tabular} & 0.5 & 1 & 2 & 4 & 8 & $>8$ & present & \\
\hline \begin{tabular}{l|l} 
Neg. &
\end{tabular} & 582 & 39 & 26 & 18 & 30 & 31 & 28 & 284 & 1038 & & 1 & & & & & 1 & 2 & 1040 \\
\hline Pos. & 1 & 2 & 3 & 1 & 2 & & & 6 & 15 & & 4 & 12 & 40 & 38 & 8 & 65 & 167 & 182 \\
\hline Sum & 583 & 41 & 29 & 19 & 32 & 31 & 28 & 290 & 1053 & & 5 & 12 & 40 & 38 & 8 & 66 & 169 & 1222 \\
\hline & CPO clas & s D car & bapen & mase & CARB & detec & & & & & & & & & & & & \\
\hline & CARBD & absent & & & & & & & & CARB & D pre & & & & & & & \\
\hline $\begin{array}{l}\text { Class D } \\
\text { carbape-nemase }\end{array}$ & Temocill & in MIC & (CPO & ested & & & & & & Temoc & illin $N$ & IC $(C$ & tis & & & & & \\
\hline genotype & & & & $\leq 4$ & 8 & 16 & 32 & $>32$ & absent & & & & & & & $>32$ & present & Sum \\
\hline Neg. & & & & 82 & 228 & 299 & 188 & 241 & 1038 & & & & & & & 2 & 2 & 1040 \\
\hline Pos. & & & & 1 & & & & 14 & 15 & & & & & & & 167 & 167 & 182 \\
\hline sum & & & & 83 & 228 & 299 & 188 & 255 & 1053 & & & & & & & 169 & 169 & 1222 \\
\hline
\end{tabular}

Table 2. MIC distribution and CPO panel detection of (un-)classified carbapenemase phenotypes (columns) in genotyped strains (rows). 


\begin{tabular}{|l|l|l|l|l|l|l|}
\hline & \multirow{2}{*}{ Ambler class genotype present } & No. of isolates & \multicolumn{6}{l|}{ CPO detect panel phenotype } \\
\cline { 3 - 8 } & $(228)$ & CARB & CARBA & CARBB & CARBD & None \\
\hline A (only) & 165 & 62 & - & - & 1 \\
\hline A and B & $(1)$ & 1 & - & - & - & - \\
\hline A and D & $(1)$ & 1 & - & - & - & - \\
\hline B (only) & $(83)$ & 5 & - & 75 & 1 & 2 \\
\hline B and D & $(6)$ & - & - & 3 & 3 & - \\
\hline D (only) & $(175)$ & 8 & - & - & 164 & 3 \\
\hline None & $(728)$ & 76 & 15 & 10 & 1 & 626 \\
\hline
\end{tabular}

Table 3. Summarized data on various phenotype results of different Ambler classes tested.

Of note, assignment to class D enzymes was made with high sensitivity, although according to other technical studies a considerable proportion of overlooked carbapenemases can be assumed due to known limitations in routine diagnostics ${ }^{32-34}$. A limitation of this study is that it does not include all difficult-to-detect OXA carbapenemases, such as the OXA-244 carbapenemases ${ }^{35}$.

One study reported a sensitivity of only $69 \%$ in the classification of class B beta-lactamases ${ }^{27}$, mainly because 28 strains investigated were not detected. This was only partially confirmed by our study, with a more favourable result of $86.7 \%$ sensitivity in assignment of class B enzymes among $90 \mathrm{MBL}$-encoding strains tested from 10 countries.

As for class A positive strains, most of the studies including the one presented here, revealed a lower rate of correct assignments of different types of class A enzymes, in particular IMI ${ }^{25}, \mathrm{KPC}^{27}$, as well as $\mathrm{KPC}_{\text {and }} \mathrm{GES}^{26}$. Moreover, in our study of 230 KPC-coding strains, only 62 could be assigned to the appropriate Ambler class, although the CPO detect test indicated 167 strains to be at least positive for an unclassified carbapenemase (CARB). These isolates were from nine countries (mainly Italy with ST258/512) and included 12 multi-locus sequence typing ST (data not shown), thus excluding local peculiarities. Our data presented here offer no further explanation for this poor specificity. Clearly, this assay as delivered here does not provide a sufficient approach to detect highly resistant class A producing organisms in which the high carbapenem MIC is lowered in the presence of class A beta-lactam inhibitors, e.g., clavulanic acid.

Besides the rather limited spectrum of carbapenemases, another limitation of this carbapenemases detection study is its confinement to K. pneumoniae. To date, it is not known whether carbapenemase coding genes are less expressed in the genomic context of other Enterobacterales. Nevertheless, species with chromosomally expressed AmpC beta-lactamases, such as Enterobacter cloacae or Klebsiella aerogenes, which may interfere with phenotypic carbapenemase detection, were not included in the study presented here. Furthermore, glucosenonfermenting Gram-negative bacilli (NFB), i.e. P. aeruginosa or Acinetobacter baumannii were not investigated here either, although depending on the epidemiological setting, they may account for a smaller number of CPO than in Enterobacterales.

Our genotypic categorization relies on draft genome sequencing data, which may have missed some coding sequences or lost plasmids despite the rigorous quality control of the sequences of the strains finally included (e.g., coverage, quality values of the base calls). Finally, it could be argued that for some specific resistances, DNA sequence data alone without others, such as transcriptome data, have limited predictive value ${ }^{36}$.

In general, demonstrating improved antimicrobial therapy decision-making based on carbapenem classification with the CPO detect panel was beyond the scope of the study presented here. This analysis did not even resistance data on relevant antibiotics, such as combinations of beta-lactamase inhibitors with carbapenems or monobactams. Further investigations are required to demonstrate a predictive value of Ambler classification in addition to antimicrobial susceptibility testing for clinical decision and outcome.

In summary, the test showed good sensitivity but lower specificity in detecting non-specific carbapenemases, with a considerable false positive rate.

This could lead to overestimation of CPO in clinical routine with all its consequences. Classification of carbapenemases employing the $\mathrm{CPO}$ detect panel resulted in accurate assignment to Ambler classes B and D. However, many isolates with class A enzymes were detected as unspecified CPO only, without a more accurate assignment to class A.

The overall sensitivity displayed by these tests in detecting any carbapenemase regardless of a more accurate classification was $98.8 \%$, which appears acceptable for routine use.

However, the comparatively low specificity of $86 \%$ leads to frequent false positive detection of carbapenemase producing organisms. Previous studies were mainly based on local strain collections of predominantly resistant strains and only a small number of sensitive strains. The low specificity displayed by the CPO test (55.3-68.6\%), also shown by other studies, was based in all cases on a small number of CPO-negative strains, ranging from 30 to 51 .

While the test was acceptable in the detection and classification of B and D carbapenemases with a sensitivity of $90-100 \%$, all the studies showed low sensitivity in the detection and classification of A carbapenemases with locally different predominating class A type enzymes. 
This CPO detect test has two main limitations in overall specificity and classification of class A carbapenemases. The low sensitivity shown in detection of class A enzymes primarily affects the assignment to this class, but not the identification of carbapenemase activity per se.

A negative $\mathrm{CPO}$ detect test result is of direct practical use/predictive value, while a positive result requires further confirmatory testing.

\section{Methods}

Strains. Sequence data on the presence/absence of carbapenemase resistance genes and multilocus sequence type were available for a set of $1222 \mathrm{~K}$. pneumoniae isolates from 1356 Enterobacterales based on draft genome sequence data. Isolates were recultivated overnight at $35^{\circ} \mathrm{C}$ on Columbia $5 \%$ sheep blood agar from a Microbank strain collection.

EUSCAPE AST data. All strains were extensively phenotypically characterized by the Italian National Reference Laboratory, Universita deligi Studi Firence, Dipartimento di Medicina Sperimentale e Clinica, Firence, Italy (Prof. Gianni Rossolini). In the latter investigation, a custom-made, semi-automated broth microdilution was used (Thermo Fischer Scientific, Basingstoke, Hampshire, UK). All isolates were tested against 16 antibiotic compounds of clinical relevance and 15 could be clinically categorized according to the European Committee on Antimicrobial Susceptibility Testing ${ }^{37}$.

EuSCAPE sequencing data. K. pneumoniae isolates were genetically characterized by high-quality whole genome sequencing carried out at the Wellcome Trust Sanger Institute, Wellcome Trust Genome Campus, Hinxton, UK. All sequence data are deposited at the European Nucleotide Archive (http://www.ebi.ac.uk/ena/data/ view/PRJEB10018) and have been published previously ${ }^{31,38}$. All reads have been mapped to relevant reference genomes and also de-novo assembled to reconstruct full draft genomes. Phylogenetic analyses of these genomes illustrate the genetic population structure of this species in Europe.

Isolate preparation for the CPO detect test and QC. Bacterial cells were resuspended in BD Phoenix AP ID Broth and adjusted to McFarland of 0.25 by use of a BD Phoenix AP instrument (Automated Nephelometry for the BD Phoenix ID/AST System). The suspension was inoculated into the BD Phoenix NMIC-502 panel, including the CPO Detect test (in short „the CPO panel”) and placed in a BD Phoenix M50 station.

Antimicrobial testing results and carbapenemase-producer classification were interpreted using the BD EpiCenter Microbiology Data Management System (V7.20/V6.35A) with breakpoints set by EUCAST ${ }^{37}$.

In general, the CPO detect test provides four different mutually exclusive data outputs. Carbapenemasephenotypes were either classified as carbapenemase-producer without further details (CARB) or phenotypically assigned to Ambler class A (CARBA), class B (CARBB) or class D (CARBD). In addition, all four carbapenemases phenotypes were grouped together as CARB_OR in this study. The electronic data were captured from the EpiCenter System and exported as a tab delimited file from the corresponding MySQL database using a script provided by BD.

Quality control was carried out on a weekly basis (E. coli ATCC 25922, P. aeruginosa ATCC 27853, E. coli ATCC 35218, K. pneumoniae ATCC 700603 or K. pneumoniae ATCC BAA-1705), as recommended by the manufacturer. If the QC control failed again when the isolate was retested, the isolate in question was not considered further in this study.

The MIC values determined by the EuSCAPE reference method were clinically categorized according to EUCAST $^{37}$ and in accordance with the BD EpiCenter Microbiology Data Management System settings mentioned above. In case of discrepant categorical results between the NMIC-502 CPO detect panel and the previously determined EuSCAPE AST data of the collection, i.e., (very) major errors, the Phoenix runs were repeated, except for colistin test results. For remaining discrepancies that could not be resolved even in a repeat run, susceptibility was then determined in dilution test procedures ${ }^{39}$.

Received: 21 January 2021; Accepted: 3 June 2021

Published online: 23 June 2021

\section{References}

1. Logan, L. K. \& Weinstein, R. A. The epidemiology of carbapenem-resistant Enterobacteriaceae: The impact and evolution of a global menace. J. Infect. Dis. 215, S28-S36. https://doi.org/10.1093/infdis/jiw282 (2017).

2. van Duin, D. \& Doi, Y. The global epidemiology of carbapenemase-producing Enterobacteriaceae. Virulence 8, 460-469. https:// doi.org/10.1080/21505594.2016.1222343 (2017).

3. Tzouvelekis, L. S., Markogiannakis, A., Piperaki, E., Souli, M. \& Daikos, G. L. Treating infections caused by carbapenemaseproducing Enterobacteriaceae. Clin. Microbiol. Infect. 20, 862-872. https://doi.org/10.1111/1469-0691.12697 (2014).

4. Tsai, Y. K. et al. Klebsiella pneumoniae outer membrane porins OmpK35 and OmpK36 play roles in both antimicrobial resistance and virulence. Antimicrob. Agents Chemother. 55, 1485-1493. https://doi.org/10.1128/AAC.01275-10 (2011).

5. Pantel, A. et al. Modulation of membrane influx and efflux in Escherichia coli Sequence type 131 has an impact on bacterial motility, biofilm formation, and virulence in a Caenorhabditis elegans model. Antimicrob. Agents Chemother. 60, 2901-2911. https://doi. org/10.1128/AAC.02872-15 (2016).

6. Ferenci, T. \& Phan, K. How porin heterogeneity and trade-offs affect the antibiotic susceptibility of gram-negative bacteria. Genes 6, 1113-1124. https://doi.org/10.3390/genes6041113 (2015).

7. Livermore, D. M., Nicolau, D. P., Hopkins, K. L. \& Meunier, D. 'CRE, CRO, CPE and CPO': Terminology past its 'sell-by-date' in an era of new antibiotics and regional carbapenemase epidemiology. Clin. Infect. Dis. https://doi.org/10.1093/cid/ciaa122 (2020).

8. Reck, F. et al. Optimization of novel monobactams with activity against carbapenem-resistant Enterobacteriaceae-Identification of LYS228. Bioorg. Med. Chem. Lett. 28, 748-755. https://doi.org/10.1016/j.bmcl.2018.01.006 (2018). 
9. Felici, A. et al. An overview of the kinetic parameters of class B beta-lactamases. Biochem. J. 291(Pt 1), 151-155. https://doi.org/ $10.1042 /$ bj2910151 (1993).

10. Nordmann, P., Poirel, L. \& Dortet, L. Rapid detection of carbapenemase-producing Enterobacteriaceae. Emerg. Infect. Dis. 18, 1503-1507. https://doi.org/10.3201/eid1809.120355 (2012).

11. Pierce, V. M. et al. Modified carbapenem inactivation method for phenotypic detection of carbapenemase production among Enterobacteriaceae. J. Clin. Microbiol. 55, 2321-2333. https://doi.org/10.1128/JCM.00193-17 (2017).

12. Carvalhaes, C. G., Picao, R. C., Nicoletti, A. G., Xavier, D. E. \& Gales, A. C. Cloverleaf test (modified Hodge test) for detecting carbapenemase production in Klebsiella pneumoniae: Be aware of false positive results. J. Antimicrob. Chemother. 65, 249-251. https://doi.org/10.1093/jac/dkp431 (2010).

13. Anderson, K. F. et al. Evaluation of methods to identify the Klebsiella pneumoniae carbapenemase in Enterobacteriaceae. J. Clin. Microbiol. 45, 2723-2725. https://doi.org/10.1128/JCM.00015-07 (2007).

14. Tsakris, A. et al. A simple phenotypic method for the differentiation of metallo-beta-lactamases and class A KPC carbapenemases in Enterobacteriaceae clinical isolates. J. Antimicrob. Chemother. 65, 1664-1671. https://doi.org/10.1093/jac/dkq210 (2010).

15. van Dijk, K. et al. A disc diffusion assay for detection of class A, B and OXA-48 carbapenemases in Enterobacteriaceae using phenyl boronic acid, dipicolinic acid and temocillin. Clin. Microbiol. Infect. 20, 345-349. https://doi.org/10.1111/1469-0691.12322 (2014).

16. Wareham, D. W., Shah, R., Betts, J. W., Phee, L. M. \& Momin, M. H. Evaluation of an immunochromatographic lateral flow assay (OXA-48 K-SeT) for rapid detection of OXA-48-like carbapenemases in Enterobacteriaceae. J. Clin. Microbiol. 54, 471-473. https:// doi.org/10.1128/JCM.02900-15 (2016).

17. Glupczynski, Y. et al. Prospective evaluation of the OKN K-SeT assay, a new multiplex immunochromatographic test for the rapid detection of OXA-48-like, KPC and NDM carbapenemases. J. Antimicrob. Chemother. 72, 1955-1960. https://doi.org/10.1093/jac/ dkx089 (2017).

18. Kaase, M., Szabados, F., Wassill, L. \& Gatermann, S. G. Detection of carbapenemases in Enterobacteriaceae by a commercial multiplex PCR. J. Clin. Microbiol. 50, 3115-3118. https://doi.org/10.1128/JCM.00991-12 (2012).

19. Monteiro, J., Widen, R. H., Pignatari, A. C., Kubasek, C. \& Silbert, S. Rapid detection of carbapenemase genes by multiplex realtime PCR. J. Antimicrob. Chemother. 67, 906-909. https://doi.org/10.1093/jac/dkr563 (2012).

20. Cunningham, S. A., Vasoo, S. \& Patel, R. Evaluation of the check-points check MDR CT103 and CT103 XL microarray kits by use of preparatory rapid cell lysis. J. Clin. Microbiol. 54, 1368-1371. https://doi.org/10.1128/JCM.03302-15 (2016).

21. Woodford, N., Warner, M., Pike, R. \& Zhang, J. Evaluation of a commercial microarray to detect carbapenemase-producing Enterobacteriaceae. J. Antimicrob. Chemother. 66, 2887-2888. https://doi.org/10.1093/jac/dkr374 (2011).

22. Koser, C. U. et al. Routine use of microbial whole genome sequencing in diagnostic and public health microbiology. PLoS Pathog. 8, e1002824. https://doi.org/10.1371/journal.ppat.1002824 (2012).

23. Pecora, N. D. et al. Genomically informed surveillance for carbapenem-resistant Enterobacteriaceae in a health care system. $m B i o$ 6, e01030. https://doi.org/10.1128/mBio.01030-15 (2015).

24. Mathers, A. J. et al. Klebsiella pneumoniae carbapenemase (KPC)-producing K. pneumoniae at a single institution: Insights into endemicity from whole-genome sequencing. Antimicrob. Agents Chemother. 59, 1656-1663. https://doi.org/10.1128/AAC.04292-14 (2015).

25. Ong, C. H., Ratnayake, L., Ang, M. L. T., Lin, R. T. P. \& Chan, D. S. G. Diagnostic accuracy of BD Phoenix CPO detect for carbapenemase production in 190 Enterobacteriaceae isolates. JClin Microbiol 56, e01043-e11018. https://doi.org/10.1128/JCM.01043-18 (2018).

26. Simon, M. et al. Evaluation of the automated BD Phoenix CPO Detect panel in combination with the beta-CARBA assay for detection and classification of carbapenemase-producing Enterobacterales. J. Microbiol. Methods 156, 29-33. https://doi.org/10. 1016/j.mimet.2018.11.024 (2018).

27. Thomson, G. et al. High-stringency evaluation of the automated BD Phoenix CPO detect and Rapidec Carba NP tests for detection and classification of carbapenemases. J. Clin. Microbiol. 55, 3437-3443. https://doi.org/10.1128/jcm.01215-17 (2017).

28. Whitley, V. et al. Multicenter evaluation of the BD Phoenix CPO detect test for detection and classification of carbapenemaseproducing organisms in clinical isolates. J. Clin. Microbiol. 58, e01752-19. https://doi.org/10.1128/JCM.01752-19 (2020).

29. Croxatto, A. et al. Evaluation of the BD Phoenix CPO detect test for the detection of carbapenemase producers. Clin Microbiol Infect 26(644), e649-e644.e615. https://doi.org/10.1016/j.cmi.2019.10.002 (2020).

30. Cho, H. et al. Performance evaluation of automated BD Phoenix NMIC-500 panel for carbapenemase detection in carbapenemresistant and carbapenem-susceptible Enterobacterales. J. Microbiol. Methods 177, 106042. https://doi.org/10.1016/j.mimet.2020. $106042(2020)$.

31. David, S. et al. Epidemic of carbapenem-resistant Klebsiella pneumoniae in Europe is driven by nosocomial spread. Nat. Microbiol. 4, 1919-1929. https://doi.org/10.1038/s41564-019-0492-8 (2019).

32. Doyle, D. et al. Laboratory detection of Enterobacteriaceae that produce carbapenemases. J. Clin. Microbiol. 50, 3877-3880. https:// doi.org/10.1128/jcm.02117-12 (2012).

33. Woodford, N. et al. Comparison of BD Phoenix, Vitek 2, and MicroScan automated systems for detection and inference of mechanisms responsible for carbapenem resistance in Enterobacteriaceae. J. Clin. Microbiol. 48, 2999-3002. https://doi.org/10.1128/jcm. 00341-10 (2010).

34. Viau, R. et al. Intestinal carriage of carbapenemase-producing organisms: Current status of surveillance methods. Clin. Microbiol. Rev. 29, 1-27. https://doi.org/10.1128/CMR.00108-14 (2016).

35. Hoyos-Mallecot, Y. et al. OXA-244-producing Escherichia coli isolates, a challenge for clinical microbiology laboratories. Antimicrob. Agents Chemother. 61, e00818-17. https://doi.org/10.1128/AAC.00818-17 (2017).

36. Khaledi, A. et al. Predicting antimicrobial resistance in Pseudomonas aeruginosa with machine learning-enabled molecular diagnostics. EMBO Mol. Med. 12, e10264. https://doi.org/10.15252/emmm.201910264 (2020).

37. EUCAST. EUCAST Breakpoint tables for interpretation of MICs and zone diameters Version 9.0, January 1, 2019. http://www. eucast.org/clinical_breakpoints/ (2019). http://www.eucast.org/clinical_breakpoints/.

38. Glasner, C. et al. Carbapenemase-producing Enterobacteriaceae in Europe: A survey among national experts from 39 countries, February 2013. Eur. Commun. Dis. Bull. 18, 9-15. https://doi.org/10.2807/1560-7917.es2013.18.28.20525 (2013).

39. CLSI. Methods for Dilution Antimicrobial Susceptibility Tests for Bacteria That Grow Aerobically. Approved Standard-Tenth Edition (Clinical and Laboratory Standards Institute, 2015).

\section{Acknowledgements}

The authors would like to thank Deborah Lawrie-Blum for assistance with preparation of the manuscript. This work was partly sponsored by Becton Dickinson Company, Diagnostic Division, Heidelberg, Germany. The material is original and has not been published elsewhere. The data were presented in part as a poster at the 29th European Congress of Clinical Microbiology and Infectious Diseases (ECCMID), 13 to 16 April 2019, Amsterdam, Netherlands. The EuSCAPE study Working Group is thanked for its contribution and comprised the following collaborators: Sophia David, Silvia Argimon, Khalil Abudahab, Richard Goater, David M. Aanensen (Centre for Genomic Pathogen Surveillance, Wellcome Genome Campus, Cambridge, UK); Sandra Reuter, Hajo 
Grundmann (Institute for Infection Prevention and Hospital Epidemiology, Faculty of Medicine, University of Freiburg, Freiburg, Germany); Simon R. Harris, Theresa Feltwell (Pathogen Genomics, Wellcome Sanger Institute, Cambridge, UK); Corinna Glasner, Hajo Grundmann (Department of Medical Microbiology and Infection Prevention, University Medical Center Groningen, University of Groningen, Groningen, The Netherlands); Tommaso Giani, Gian Maria Rossolini (Department of Experimental and Clinical Medicine, University of Florence, Florence, Italy); Giulia Errico (Department of Infectious Diseases, Istituto Superiore di Sanità, Rome, Italy); Marianne Aspbury (Natural Sciences, University of Bath, Bath, UK); Sara Sjunnebo (Pathogen Informatics, Wellcome Sanger Institute, Cambridge, UK); Edward J. Feil (Milner Centre for Evolution, Department of Biology and Biochemistry, University of Bath, Bath, UK); Gian Maria Rossolini (Clinical Microbiology and Virology Unit, Florence Careggi University Hospital, Florence, Italy); David M. Aanensen (Big Data Institute, Li Ka Shing Centre for Health Information and Discovery, Nuffield Department of Medicine, University of Oxford, Oxford, UK); Andi Koraqi, Denada Lacej (University Hospital Center Mother Theresa, Tirana, Albania); Petra Apfalter, Rainer Hartl (Elisabethinen Hospital Linz, Linz, Austria); Youri Glupczynski, Te-Din Huang (CHU DinantGodinne, UCL Namur, Namur, Belgium); Tanya Strateva (Faculty of Medicine, Medical University of Sofia, Sofia, Bulgaria); Yuliya Marteva-Proevska (Alexandrovska University Hospital, Sofia, Bulgaria); Arjana Tambic Andrasevic, Iva Butic (University Hospital for Infectious Diseases, Zagreb, Croatia); Despo Pieridou-Bagatzouni, Panagiota Maikanti-Charalampous (Nicosia General Hospital, Nicosia, Cyprus); Jaroslav Hrabak (Faculty of Medicine in Plzen, Charles University in Prague, Plzen, Czech Republic); Helena Zemlickova (National Institute of Public Health, Prague, Czech Republic); Anette Hammerum, Lotte Jakobsen (Statens Serum Institut, Copenhagen, Denmark); Marina Ivanova, Anastasia Pavelkovich (East Tallinn Central Hospital, Tallinn, Estonia); Jari Jalava, Monica Österblad (National Institute for Health and Welfare, Turku, Finland); Laurent Dortet (Bicêtre Hospital, Le Kremlin-Bicêtre, France); Sophie Vaux (Institut de Veille Sanitaire, Saint-Maurice, France); Martin Kaase (Universitätsmedizin Göttingen, Göttingen, Germany); Sören G. Gatermann (Ruhr-University Bochum, Bochum, Germany); Alkiviadis Vatopoulos, Kyriaki Tryfinopoulou (National School of Public Health, Athens, Greece); Ákos Tóth, Laura Jánvári (National Center for Epidemiology, Budapest, Hungary); Teck Wee Boo, Elaine McGrath (Galway University Hospitals, Galway, Ireland); Yehuda Carmeli, Amos Adler (Ministry of Health, Tel Aviv, Israel); Annalisa Pantosti, Monica Monaco (Istituto Superiore di Sanità, Rome, Italy); Lul Raka, Arsim Kurti (National Institute of Public Health of Kosovo, Prishtina, Kosovo); Arta Balode, Mara Saule (Pauls Stradins Clinical University Hospital, Riga, Latvia); Jolanta Miciuleviciene, Aiste Mierauskaite (National Public Health Surveillance Laboratory, Vilnius, Lithuania); Monique Perrin-Weniger, Paul Reichert (Laboratoire National De Sante, Düdelingen, Luxembourg); Nina Nestorova, Sonia Debattista (Mater Dei Hospital, Msida, Malta); Gordana Mijovic, Milena Lopicic (Institute of Public Health, Podgorica, Montenegro); Ørjan Samuelsen, Bjørg Haldorsen (University Hospital of North Norway, Tromsø, Norway); Dorota Zabicka (Narodowy Instytut Lekow, Warsaw, Poland); Elzbieta Literacka (National Medicines Institute, Warsaw, Poland); Manuela Caniça, Vera Manageiro (National Institute of Health Dr. Ricardo Jorge, Lisbon, Portugal); Ana Kaftandzieva, Elena Trajkovska-Dokic (Medical Faculty, Institute of Microbiology and Parasitology, Skopje, Republic of Macedonia); Maria Damian, Brandusa Lixandru (Cantacuzino National Research Institute, Bucharest, Romania); Zora Jelesic (Institute for Public Health of Vojvodina, Novi Sad, Serbia); Anika Trudic (Institute for Pulmonary Diseases of Vojvodina, Sremska Kamenica, Serbia); Milan Niks (Public Health Authority of the Slovak Republic, Bratislava, Slovakia); Eva Schreterova (Faculty of Medicine, P. J. Šafárik University, Kosice, Slovakia); Mateja Pirs, Tjasa Cerar (Institute of Microbiology and Immunology, Ljubljana, Slovenia); Jesús Oteo, Belén Aracil (Centro Nacional de Microbiología, Instituto de Salud Carlos III, Madrid, Spain); Christian Giske (Karolinska Institutet, Stockholm, Sweden); Karin Sjöström (Public Health Agency of Sweden, Stockholm, Sweden); Deniz Gür, Asli Cakar (Hacettepe University, Ankara, Turkey); Neil Woodford, Katie Hopkins (Reference Microbiology Services, Public Health England, London, UK); Camilla Wiuff (Sydvestjysk Hospital, Esbjerg, Denmark); Derek J. Brown (Scottish Microbiology Reference Laboratories, Glasgow, UK).

\section{Author contributions}

Conceived and designed the experiments: D.J., and H.G. Performed the experiments: S.K., S.W., and M.B. Analyzed the data: D.J., S.R., T.G., and G.M.R.. Wrote the paper: D.J., G.M.R., and H.G.

\section{Funding}

Open Access funding enabled and organized by Projekt DEAL.

\section{Competing interests}

The authors declare no competing interests.

\section{Additional information}

Correspondence and requests for materials should be addressed to D.J.

Reprints and permissions information is available at www.nature.com/reprints.

Publisher's note Springer Nature remains neutral with regard to jurisdictional claims in published maps and institutional affiliations. 
(c) (i) Open Access This article is licensed under a Creative Commons Attribution 4.0 International cc) License, which permits use, sharing, adaptation, distribution and reproduction in any medium or format, as long as you give appropriate credit to the original author(s) and the source, provide a link to the Creative Commons licence, and indicate if changes were made. The images or other third party material in this article are included in the article's Creative Commons licence, unless indicated otherwise in a credit line to the material. If material is not included in the article's Creative Commons licence and your intended use is not permitted by statutory regulation or exceeds the permitted use, you will need to obtain permission directly from the copyright holder. To view a copy of this licence, visit http://creativecommons.org/licenses/by/4.0/.

(C) The Author(s) 2021 